\title{
Les technologies au service des étudiants vivant avec un \\ handicap invisible
}

doi:10.18162/fp.2015.d66

\section{CHRONIQUE • Intervention éducative}

S'appuyant sur des écrits scientifiques ainsi que sur notre expérience à titre de responsable des technologies adaptées à l'Université Laval, le présent article s'intéresse aux aides technologiques permettant aux étudiants en situation de troubles d'apprentissage (TA) de poursuivre avec succès leur cheminement scolaire postsecondaire.

\section{Des handicaps invisibles chez un nombre croissant d'étu- diants}

Les TA se manifestent principalement par des difficultés en lectureécriture de type dyslexie ou dysorthographie. On les appelle handicaps invisibles, car ils sont sans manifestation physique évidente. Les étudiants vivant avec un handicap invisible se retrouvent dans une situation délicate. Ces étudiants

[...] restent longtemps dans l'ombre, ne voulant pas s'exposer, qui par souci d'anonymat, qui par peur de la stigmatisation. C'est souvent après plusieurs obstacles, des difficultés scolaires importantes, et parfois quelques échecs, qu'ils se risquent à divulguer leur trouble pour pouvoir obtenir de l'aide. (Raymond, 2012, p. 5)

Or, les étudiants vivant avec un handicap invisible constituent une population en croissance dans les établissements postsecondaires. En milieu universitaire, par exemple, leur nombre a augmenté de $143 \%$ pour atteindre en 2012 plus de 2400 étudiants (Conférence des recteurs et des principaux des universités du Québec [CREPUQ], 2012). Cela pose des défis particuliers aux centres d'aide, qui reçoivent plusieurs nouveaux étudiants chaque année et doivent 
répondre aux demandes d'aide pour l'achat et l'utilisation de matériel adapté, ainsi qu'aux demandes d'accommodement pour la prise de notes, le tutorat et la passation d'examens.

\section{Les technologies d'assistance, un aperçu}

Les technologies d'assistance regroupent les logiciels spécialisés qui pallient, entre autres, les difficultés en lecture-écriture. Parmi les logiciels d'aide à la lecture-écriture, nous retrouvons les correcteurs orthographiques, les prédicteurs vocaux, qui suggèrent des mots à partir des premières lettres tapées, les synthèses vocales, qui lisent le texte à l'écran, et les cartes conceptuelles, qui aident à organiser les idées.

En plus de pallier les difficultés en lecture et en écriture, les technologies d'assistance favorisent l'autonomie et rendent possible la personnalisation des apprentissages. Les utilisateurs sont ainsi en mesure de lire des textes universitaires complexes, de prendre leurs propres notes durant les cours, plutôt que de dépendre d'autres étudiants engagés pour le faire, et de rendre des travaux de meilleure qualité. Selon Grant (2008), les logiciels apportent aux étudiants une plus grande indépendance, un plus grand engagement dans leurs études, entre autres en mettant de l'avant leurs points forts. Ainsi, les technologies d'assistance rendent les études accessibles et les réussites possibles.

\section{Au-delà de l'aide en lecture-écriture}

Par ailleurs, les personnes vivant avec un TA ont souvent une faible estime de soi et vivent davantage de détresse que les étudiants sans TA (Heiman et Shemesh, 2011). Les technologies d'assistance leur permettent de percevoir plus positivement leur cheminement scolaire (Alkahtani, 2013). Elles peuvent aussi changer la perception que ces personnes ont d'eux-mêmes. «Un changement de perception des jeunes à l'égard de leurs propres capacités intellectuelles constituerait un atout important pour faciliter leur réussite jusqu'à l'obtention d'un diplôme »(Teixeira, 2013, p. 48).

Les réussites que peuvent vivre les étudiants, aidés des bons outils et accompagnés par les centres d'aide de leur établissement d'enseignement, les amènent en effet à se sentir compétents. "The students participants reported elevated academic self-perception specific to reading comprehension and pronouncing (speaking), and wanted to read more » (Chiang et Jacobs, 2010, p. 353). Cela favorise la mise en place du « cercle vertueux » dont parle Teixeira (2013) qui s'oppose au cercle vicieux de l'échec et du décrochage scolaire.

\section{Une difficile acceptation}

Même une fois le diagnostic posé, des doutes subsistent toutefois dans les milieux éducatifs au sujet de l'usage des technologies adaptées. Dans son mémoire sur l'encadrement de la clientèle émergente, Marie-Christine Dion (2013, p. 127) cite un intervenant : "Je ne sais pas jusqu'à quel point on les aide. [...] Dans son milieu de travail, il n'aura pas accès à ces outils ». D'une part, dans un contexte universitaire où les documents à lire sont particulièrement nombreux et volumineux et les travaux rapprochés dans le temps, les étudiants ont besoin d'une aide rapidement efficace. D'autre part, notre expérience montre qu'il n'est pas tout à fait juste de dire que l'étudiant n'aura pas accès à ces outils en milieu de travail. Au contraire, s'il les maitrise bien, il peut souvent les intégrer dans son emploi. 
Par ailleurs, nous rencontrons aussi dans notre pratique des enseignants qui soulèvent des questions d'équité, par exemple dans les cas où les étudiants obtiennent des résultats élevés lorsqu'ils ont recours à du matériel adapté. C'est pourtant le rôle des accommodements de pallier le TA des étudiants pour qu'ils puissent atteindre leur plein potentiel. Cela serait, il nous semble, à accueillir favorablement.

\section{Conclusion}

Les technologies d'assistance atténuent les difficultés en lecture-écriture, mais permettent également une plus grande autonomie et une meilleure estime de soi. Dans un monde idéal, les jeunes se familiariseraient tôt avec ces technologies. Cependant, dans la réalité, plusieurs d'entre eux reçoivent un diagnostic tardif et doivent apprendre à utiliser les technologies d'assistance au collège ou à l'université. Il est important que les établissements d'enseignement forment des membres de leur personnel afin qu'ils soient en mesure de bien conseiller ces étudiants et de leur fournir des aides technologiques faciles à utiliser et rapidement accessibles. Il est également important de sensibiliser les enseignants, les conseillers pédagogiques et l'ensemble des étudiants à l'utilité des technologies adaptées et à l'équité de leur usage.

\section{Références}

Alkahtani, K. D. F. (2013). Teachers' knowledge and use of assistive technology for students with special educational needs. Journal of Studies in Education, 3(2), 65-86. http://dx.doi.org/10.5296/jse.v3i2.3424

Chiang, H. Y. et Jacobs, K. (2010). Perceptions of a computer-based instruction system in special education: high school teachers and students views. Work, 37(4), 349-359. http://dx.doi.org/10.3233/WOR-2010-1089

Conférence des recteurs et des principaux des universités du Québec (CREPUQ). (2012). Position des établissements universitaires à l'égard de l'intégration des clientèles émergentes à l'université.

Dion, M.-C. (2013). Représentations sociales des enseignantes et des enseignants des secteurs collégial et universitaire du SaguenayLac-Saint-Jean quant au soutien et à l'encadrement de la "clientèle émergente » (mémoire de maîtrise, Université du Québec à Chicoutimi, Canada). Repéré à http://constellation.uqac.ca/2765/

Grant, K. (2008). Inclusive technology - tools for learning. Special Education Technology Practice, 10(4), 27-32.

Heiman, T. et Shemesh, D. O. (2011). Students with learning disabilities in higher education : use and contribution of assistive technology and website courses and their correlation to students' hope and well-being. Journal of Learning Disabilities, 45(4), 308-318. http://dx.doi.org/10.1177/0022219410392047

Raymond, O. (2012). L’inclusion, d'hier à demain. Pédagogie collégiale, 25(4), 4-8. Repéré à http://www.cdc.qc.ca/ped coll/v25/Raymond-inclusion-25-4-2012.pdf

Teixeira, M. (2013). Réussite scolaire et action dialogique selon Paulo Freire : la conscience de la propre capacité intellectuelle chez les élèves à risque de décrochage au secondaire. Initio, (Réussir et soutenir la réussite : regards croisés des sciences de l'éducation, hors-série 1), 47-62. Repéré à http://www.initio.fse.ulaval.ca/fichiers/site initio/documents/ Hors Serie 1 - ACCESE/Teixeira INITIO no. H-S ete 2013.pdf

\section{Pour citer cet article}

Gagné, Y. (2015). Les technologies au service des étudiants vivant avec un handicap invisible. Formation et profession, 23(3), 117-119. http://dx.doi.org/10.18162/fp.2015.a66 